\title{
Molecular dissection of plant height QTLs using recombinant inbred lines from hybrids between common wheat (Triticum aestivum L.) and spelt wheat (Triticum spelta L.)
}

\author{
LIU Gang, XU ShengBao, NI ZhongFu, XIE ChaoJie, QIN DanDan, LI Jing, LU LaHu, \\ ZHANG JinPing, PENG HuiRu ${ }^{*} \&$ SUN QiXin ${ }^{*}$
} State Key Laboratory for Agrobiotechnology, College of Agronomy and Biotechnology, Key Laboratory of Crop Genomics and Genetic Improve-
ment of Ministry of Agriculture, Beijing Key Laboratory of Crop Genetic Improvement, Key Laboratory of Crop Heterosis Research and Utiliza-
tion of Ministry of Education, China Agricultural University, Beijing 100193, China

Received November 29, 2010; accepted April 2, 2011; published online May 12, 2011

\begin{abstract}
In wheat, plant height is an important agronomic trait, and a number of quantitative trait loci (QTLs) controlling plant height have been located. In this study, using the conditional and unconditional QTL mapping methods, combined with data from five different growth stages over two years of field trials, the developmental behavior for plant height in wheat was dissected. Nine unconditional QTLs and 8 conditional QTLs were identified, of which 6 were detected by both methods. None of the 11 QTLs was detected at all of the 5 investigated developmental stages, but 7 QTLs were detected at certain stages in both years. Further analysis identified 9 unconditional QTLs at different stages, which could explain the phenotypic variation from $4.81 \%$ to $17.35 \%$. It was noteworthy that one major QTL designated $Q H t-4 B-2$, which was located on chromosome 4B, was detected on May 18 and 25 in both years, and its genetic contributions to plant height ranged from $13.42 \%$ to $16.13 \%$. Moreover, of the 8 conditional QTLs identified, six were detected in both years, in the order of $Q H t-3 B \rightarrow Q H t-4 B-1 \rightarrow Q H t-4 B-2 \rightarrow Q H t-4 D \rightarrow Q H t-5 A$ and $Q H t-2 B$ expressed at the same developmental stage. The results indicate that QTL expression during plant height development is selective and in a temporal order.
\end{abstract}

wheat, plant height, conditional QTL, unconditional QTL

Citation: Liu G, Xu S B, Ni Z F, et al. Molecular dissection of plant height QTLs using recombinant inbred lines from hybrids between common wheat (Triticum aestivum L.) and spelt wheat (Triticum spelta L.). Chinese Sci Bull, 2011, 56: 1897-1903, doi: 10.1007/s11434-011-4506-Z

As one of the most important agronomic traits in wheat breeding, plant height plays a vital role in producing plants with high lodging resistance and high harvest index. For example, the dwarfism gene from Nonglin-10, which was widely used in classical wheat breeding programs, contributed significantly to the green revolution in the 1960s [1]. Until now, dwarfism breeding has been important in increasing the yield of wheat all over the world.

Genetic analysis of plant height has been carried out by many researchers, and more than 20 dwarfism genes have been identified in wheat [2]. Some of these genes have been

*Corresponding authors (email: penghuiru@cau.edu.cn; qxsun@ cau.edu.cn) exploited to breed many semidwarf cultivars with high yield potential. Recently, along with the advent of molecular quantitative genetics, about 50 quantitative trait loci (QTLs) for wheat plant height have been identified using the constructed molecular genetic linkage maps [3-15]. However, QTL mapping of plant height in wheat was only focused on the performance of this trait at the maturing stage.

Conventional statistical genetic analysis revealed that gene expression patterns were distinct at different developmental stages, and the genetic model for the final character did not fully reflect the true gene expression pattern during formation of the character [16-22]. Therefore it is necessary to conduct dynamic mapping of plant height at a variety of 
stages to fully elucidate the genetic basis of the developmental behavior of the trait. Indeed, conditional and unconditional QTL mapping methods developed by Zhu [21] have already been used successfully to study the developmental behavior of plant height in other crops, such as rice [22], maize [23] and soybean [24]. These studies indicated that the actions and interactions of genes responsible for plant height varied in different growth periods and environmental conditions. Recently, QTL mapping for developmental behavior of plant height in wheat was conducted under well-watered and drought conditions [25].

Spelt (Triticum spelta L.) is a hexaploid species of wheat, an ancient and distant cousin of modern wheat (Triticum aestivum L.), and it possesses many genes valuable for conferring tolerance to biotic and abiotic stress, as well as flour quality [26], which could be useful for wheat breeding. As dwarf wheat, Line 3338 is ideal for the study of plant height and QTLs for plant height have been detected using an $\mathrm{F}_{2: 3}$ population derived from Line 3338 and F390 [27]. In the present study, a population of 188 advanced recombinant inbred lines was derived from a cross between a common wheat (T. aestivum AABBDD) Line 3338 and spelt wheat (T. spelta AABBDD) Altgold. The population was further used to analyze the developmental behavior of plant height, and the 11 conditional and unconditional QTLs of plant height detected in our study enabled us to further understand the genetic basis of plant height in wheat.

\section{Materials and methods}

\subsection{Materials and genetic maps}

A population of $188\left(\mathrm{~F}_{7}\right)$ recombinant inbred lines derived from a cross between the common wheat ( $T$. aestivum) Line 3338 and spelt wheat (T. apelta) Altgold using a single seed descent method was used for constructing a genetic linkage map and QTL mapping. Using Mapmaker/EXP version 3.0b (centimorgan function: Kosambi, LOD > 3), a genetic linkage map was constructed with 290 DNA markers (232 simple sequence repeat [SSR], 56 expressed sequence tag (EST)-SSR and two gene-specific markers), which were distributed throughout the 21 chromosomes (data not shown). This map covered $4048.3 \mathrm{cM}$ with an average distance of $13.9 \mathrm{~cm}$ (maximum $48.2 \mathrm{~cm}$, minimum $0 \mathrm{~cm}$ ) between pairs of markers. The SSR markers used in this study were taken from the GrainGenes database (http://wheat.pw. usda.gov), and the EST-SSR markers (designated as Xcau) were designed by the Wheat Genetics and Genomics Laboratory of China Agricultural University. The $Q$ gene-specific primers were designed based on the sequence variation between common wheat and spelt wheat according to Simons et al. [28]. The Rht-B1-specific marker (BF-WR1) was exploited by Ellis et al. [29].

\subsection{Measurement of plant height}

The experiment was conducted during the April, 2003 and
May, 2004 growing seasons at the Changping experimental station, Beijing, of the China Agricultural University. Seeds of the $188 \mathrm{~F}_{7}$ RILs and their parents Line 3338 and Altgold were sown on October 3 in both 2003 and 2004 in a randomized complete block design with three replications. A spacing of $20 \mathrm{~cm}$ between rows and $20 \mathrm{~cm}$ between plants was maintained within a 2-m-long row, which represented a single plot. The management of the field experiments was in accordance with local standard practices.

Starting on April 27, plant height was measured for 5 plants in each plot every $7 \mathrm{~d}$ until the height remained unchanged. Height (in $\mathrm{cm}$ ) from the soil surface to the tip of the tallest ear was considered as actual plant height (awns excluded).

\subsection{Data analysis and QTL mapping}

The unconditional QTLs for plant height were mapped using the composite interval mapping method [30]. The genetic effect detected by unconditional mapping represented the total effect of the QTL from the initial time to time $t$. The conditional phenotypic value $(y(t) / y(t-1))$ of plant height was obtained by mixed model approaches for the conditional genetics of developmental quantitative traits [21], and the composite interval mapping method was used to analyze the conditional phenotypic value. The conditional QTLs were detected by conditional mapping, which accounts for gene expression at a specific developmental pe$\operatorname{riod}(t-1$ to $t)$, and the effect of the QTL is the genetic net effect during the time interval ( $t-1$ to $t$ ). The conditional phenotypic value was calculated with QGA Station version 1.0 software. Windows QTL Cartographer version 2.5 was used to locate QTLs (LOD $\geqslant 2.5$ ).

\section{Results}

\subsection{Plant height at 5 different developmental stages}

The plant height of the 188 RILs and the parents Line 3338 and Altgold was measured at 5 different developmental stages in both growing seasons (Table 1). The male parent Altgold grew much faster and was much taller than the female parent Line 3338 at all investigated stages in both growing seasons. Line 3338 grew quickly only at the first investigated stage, namely from April 27 to May 4 (Table 1). This was probably because Line 3338 is an early heading genotype, which resulted in cessation of vegetative growth from May 4. Broad variation of plant height among the RILs existed (Table 1). At the final stage, the average plant height of the 188 RIL lines was $92.5 \mathrm{~cm}$ and ranged from 56.2 to $131.9 \mathrm{~cm}$.

\subsection{Unconditional QTL analysis}

Using unconditional plant height phenotypic values measured 
Table 1 Plant height $(\mathrm{cm})$ of the RIL population and two parents at different stages in 2004 and 2005

\begin{tabular}{|c|c|c|c|c|c|c|c|c|}
\hline \multirow{2}{*}{ Date } & \multirow{2}{*}{ Year } & \multicolumn{2}{|c|}{ Parents } & \multicolumn{5}{|c|}{ RILs } \\
\hline & & 3338 & Altgold & Min & Max & Mean & Skew & Kurt \\
\hline \multirow[t]{2}{*}{ April 27} & 2004 & $50.8 \pm 0.84$ & $54.4 \pm 2.07$ & 34 & 81.2 & $57.7 \pm 8.9$ & -0.23 & -0.12 \\
\hline & 2005 & $43.2 \pm 0.84$ & $54.6 \pm 3.44$ & 31 & 72.4 & $53.5 \pm 7.5$ & -0.01 & -0.11 \\
\hline \multirow[t]{2}{*}{ May 4} & 2004 & $58.2 \pm 1.67$ & $63.4 \pm 2.30$ & 42 & 102.8 & $71.9 \pm 11.4$ & -0.33 & -0.02 \\
\hline & 2005 & $53.4 \pm 1.41$ & $71.6 \pm 1.22$ & 41.4 & 89.8 & $67.6 \pm 9.4$ & -0.17 & -0.23 \\
\hline \multirow[t]{2}{*}{ May 11} & 2004 & $60.4 \pm 3.63$ & $80.2 \pm 3.11$ & 50 & 108.4 & $83.7 \pm 12.6$ & -0.49 & -0.08 \\
\hline & 2005 & $53.6 \pm 1.58$ & $84.8 \pm 3.51$ & 51 & 100.2 & $80.3 \pm 14.5$ & -0.45 & -0.19 \\
\hline \multirow[t]{2}{*}{ May 18} & 2004 & $60.4 \pm 0.59$ & $93 \pm 4.74$ & 51 & 126.8 & $89.1 \pm 13.3$ & -0.47 & 0.24 \\
\hline & 2005 & $52.6 \pm 1.22$ & $105.6 \pm 4.30$ & 57.2 & 127.8 & $89.1 \pm 13.2$ & -0.11 & -0.15 \\
\hline \multirow[t]{2}{*}{ May 28} & 2004 & $58.3 \pm 1.51$ & $108 \pm 5.71$ & 54.6 & 132.4 & $92.5 \pm 14.1$ & -0.38 & 0.37 \\
\hline & 2005 & $52.6 \pm 1.95$ & $119.2 \pm 5.57$ & 57.8 & 131.4 & $92.5 \pm 14.8$ & 0.02 & -0.32 \\
\hline
\end{tabular}

at 5 different stages, seven and eight QTLs were detected at different stages in 2004 and 2005, respectively (Table 2, Figure 1). Of these QTLs, 6 were detected in both years, with slight differences with growth stage and genetic effect value. For example, one QTL ( $Q H t-4 B-2)$, which explained $12.17 \%$ (2004) and $16.13 \%$ (2005) of phenotypic variation in plant height, was detected on May 11, 18 and 25 in 2004, and on May 18 and 25 in 2005. Another QTL ( $Q H t-3 B)$ was detected at the four earliest stages in 2004, but was detected only at the first stage in 2005 , and was not detected at the final stage in 2004 or 2005. Similarly, QHt-4B-1 was detected at the first two stages and at the three earliest stages in 2004 and 2005, respectively, but was not detected at the last stage. $Q H t-7 B-1$ and $Q H t-7 B-2$ were only detected in 2005 , while $Q H t-2 D$ was only detected at the last stage in 2004. It was no table that only five QTLs were detected at the last stage (May 25), and three of these were common to both growing seasons, which was much fewer than QTLs detected at the different developmental stages, suggesting that some QTLs expressed early during growth in plant height might be inactive or undetectable as the plants matured.

\subsection{Conditional QTL analysis}

Using a conditional QTL mapping method, six and eight QTLs of plant height were detected at the five different stages in 2004 and 2005, respectively, of which six were detected in both years (Table 3, Figure 1). Among the three QTLs detected at the initial stage to April 27, two were detected in both years, but none of QTLs detected from April 27 to May 4 were identical in both years. Only one QTL $(Q H t-4 D)$ was detected from May 4 to May 11 only in 2004. Though 3 QTLs were identified from May 11 to May 18 , only one locus $(Q H t-4 B-2)$ was detected in both years. At the last stage, loci $Q H t-2 B$ and $Q H t-5 A$ were detected

Table 2 Effect of unconditional QTLs for plant height at different stages in 2004 and 2005

\begin{tabular}{|c|c|c|c|c|c|c|c|c|c|c|c|c|c|c|c|c|c|}
\hline \multirow{2}{*}{ QTL } & \multirow{2}{*}{ Interval } & \multirow{2}{*}{ Year } & \multicolumn{3}{|c|}{$4-27$} & \multicolumn{3}{|c|}{$5-4$} & \multicolumn{3}{|c|}{$5-11$} & \multicolumn{3}{|c|}{$5-18$} & \multicolumn{3}{|c|}{$5-25$} \\
\hline & & & LOD & $A^{\text {a) }}$ & $R^{2} \%^{\text {b) }}$ & LOD & $A$ & $R^{2} \%$ & LOD & $A$ & $R^{2} \%$ & LOD & $A$ & $R^{2} \%$ & LOD & $A$ & $R^{2} \%$ \\
\hline \multirow[t]{2}{*}{$Q H t-2 D$} & Xcfd44-Xgwm539 & 2004 & & & & & & & & & & & & & 3.62 & 4.62 & 10.07 \\
\hline & & 2005 & & & & & & & & & & & & & & & \\
\hline \multirow[t]{2}{*}{$Q H t-3 B$} & Xgwm566-Хсаи101 & 2004 & 3.68 & 2.47 & 7.22 & 4.74 & 4.58 & 10.33 & 2.84 & 3.02 & 5.47 & 4.45 & 4.99 & 9.07 & & & \\
\hline & & 2005 & 2.56 & 1.69 & 4.81 & & & & & & & & & & & & \\
\hline$Q H t-4 A$ & Xwmc24-Xksm130 & 2004 & & & & & & & 2.55 & 3.61 & 7.96 & 2.89 & 3.16 & 5.3 & & & \\
\hline \multirow[t]{2}{*}{$Q H t-4 B-1$} & Xgwm375-Xgwm251 & 2004 & 5.77 & -3.08 & 11.00 & 6.01 & -4.05 & 12.00 & & & & & & & & & \\
\hline & & 2005 & 5.47 & -2.48 & 10.57 & 4.04 & -2.76 & 8.33 & 3.89 & -4.20 & 8.17 & & & & & & \\
\hline \multirow[t]{2}{*}{$Q H t-4 B-2$} & Xgwm495-Xgwm375 & 2004 & & & & & & & 5.76 & -4.46 & 12.17 & 7.36 & -5.43 & 16.13 & 6.58 & -5.32 & 13.8 \\
\hline & & 2005 & & & & & & & & & & 7.58 & -5.17 & 15.11 & 6.50 & -5.48 & 13.42 \\
\hline \multirow[t]{2}{*}{$Q H t-4 D$} & Xpsp3103-Xcfd71 & 2004 & & & & & & & 3.19 & -3.27 & 6.57 & 3.64 & -3.69 & 7.51 & 2.91 & -3.51 & 5.99 \\
\hline & & 2005 & & & & & & & & & & 4.53 & -4.09 & 9.47 & 4.60 & -4.92 & 10.79 \\
\hline$Q H t-5 A$ & $Q-X c f a 21559$ & 2004 & & & & & & & & & & & & & 4.27 & -4.68 & 10.62 \\
\hline \multirow[t]{2}{*}{$Q H t-7 B-1$} & Xcau130-Xgwm537 & 2004 & & & & & & & & & & & & & & & \\
\hline & & 2005 & 3.03 & 1.9 & 6.04 & 3.26 & 2.68 & 7.74 & & & & & & & & & \\
\hline \multirow[t]{2}{*}{$Q H t-7 B-2$} & Xgdm36-Xbarc50 & 2004 & & & & & & & & & & & & & & & \\
\hline & & 2005 & & & & & & & & & & 2.84 & 3.13 & 5.58 & & & \\
\hline
\end{tabular}

a) Additive effect: positive value of the additive effect that Line 3338 alleles are in the direction of increasing the plant height; b) phenotypic variation explained by each QTL. 

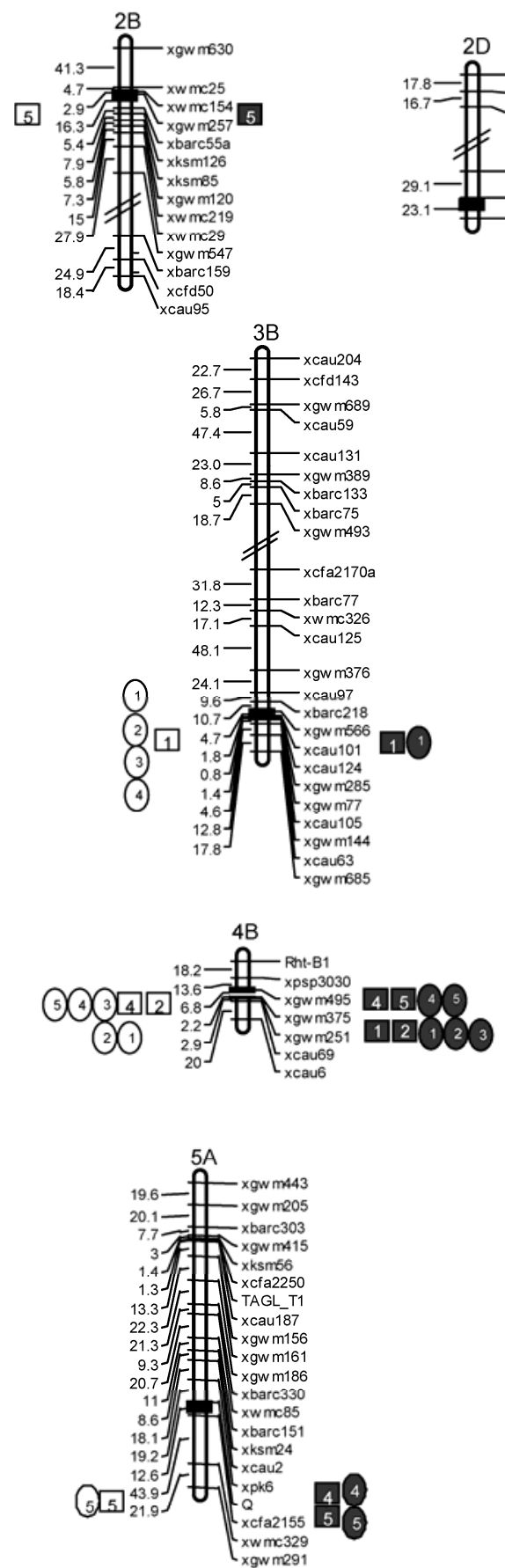

Conditional QTL at different stages in 2004

Conditional QTL at different stages in 2005 ,

O
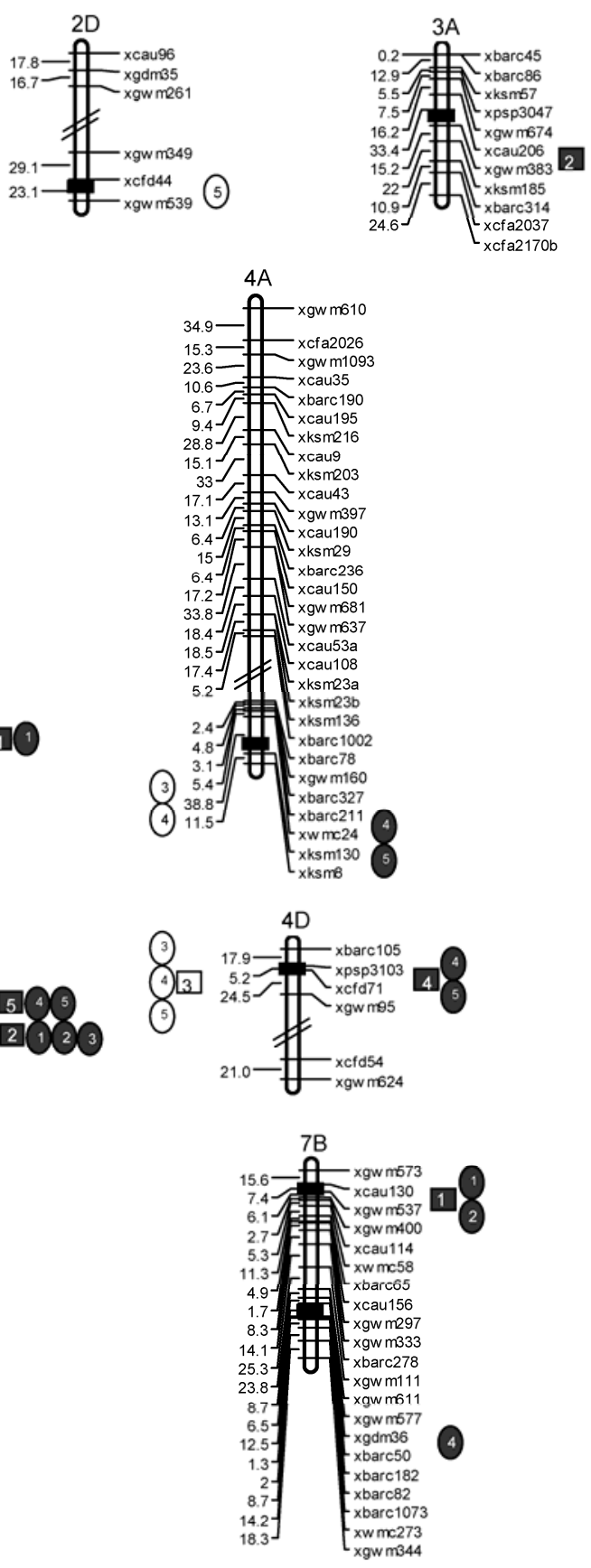

Unconditional QTL at different stages in 2005,

Position of QTL on chromosome

Number indicated the 5 different measured stages

Figure 1 Conditional and unconditional QTLs for wheat plant height at different stages.

in both years. Of all the QTLs detected at the last four stages (from April 27 to May 25), the Altgold alleles increased plant height, which was in accordance with the fact that growth of Altgold continued while Line 3338 stopped growing after April 27. The result also indicated that no QTLs/genes for plant height were continually active during the whole period of growth. Interestingly, expression of the 6 QTLs was in the order of $Q H t-3 B \rightarrow Q H t-4 B-1 \rightarrow$ $Q H t-4 B-2 \rightarrow Q H t-4 D \rightarrow Q H t-5 A$ and $Q H t-2 B$ expressed at the same developmental stage in both years. Further analysis showed that the genetic effect of these QTLs was quite similar for the two years, though the size of effects varied 
(Table 3).

\section{Discussion}

The formation of plant height is a dynamic process, involving the expression of many genes and other important regulatory mechanisms. Most previous studies related to QTL mapping of plant height have focused on the trait at the maturing stage, which might lead to loss of much important information. In order to detect loci underlying the developmental behavior of plant height in wheat, two QTL mapping methods, comprising unconditional and conditional methods, were used in the present study. The former method involves mapping QTLs using phenotypic values at each observed time point, which is referred to as accumulation analysis of QTL from the initial time to the time point $t$. The latter method identifies QTLs using increments of phenotypic values from the specific time point $t-1$ to $t$, reflecting the net effects of gene expression from time $t-1$ to $t$.

In this study, expression of QTLs/genes for wheat plant height at 5 growth stages in two years was assessed using dynamic QTL mapping methods. Nine unconditional QTLs and eight conditional QTLs responsible for plant height were identified in the RIL population derived from a cross between common wheat (T. aestivum) Line 3338 and spelt wheat (T. spelta) Altgold (Tables 2 and 3, Figure 1), respectively. More QTLs were detected at immature stages than at the maturing stage and no QTL for plant height was continually active during the whole period of growth. We also observed many differences between conditional and unconditional QTLs, for example, one unconditional QTL located on chromosome 4D and flanked by the SSR markers xpsp3103 and $x c f d 71$ was detected at three stages in 2004 (May 11, 18 and 25), but was only expressed from May 4 to May 11 during the conditional QTL analysis. In addition, two conditional QTLs $(Q H t-2 B$ and $Q H t-3 A)$ identified in the present study were not be detected in the unconditional QTL analysis. These results indicated that some of the conditional QTLs were only actively expressed at one or two stages compared to unconditional QTLs. In other words, these QTLs only play an important role at certain developmental stages. These results were in agreement with previous studies of plant height in rice [22] and maize [23], which also reflected the developmental genetics and biology theory that genes are expressed selectively at different growth stages. Therefore, the conditional QTL method enabled us to identify the important developmental stages that we should focus on, which was helpful to understand the genetic and molecular mechanisms of plant height in wheat.

Though the average plant height of the RIL population was quite different between the two years, they were identical at the last two investigated stages in both years (Table 1). In addition, plants of the female parent Line 3338 at the different stages in 2004 were taller than in 2005, while plants of the male parent Altgold in 2004 were much shorter than in 2005 (Table 1). Similar results have been reported by Wang [25]. We also found that the QTLs detected by both conditional and unconditional analysis in both years showed slight differences, such as $Q H t-3 B$, which was

Table 3 Effect of conditional QTLs $(t / t-1)$ for plant height at different stages in 2004 and $2005^{\text {a) }}$

\begin{tabular}{|c|c|c|c|c|c|c|c|c|c|c|c|c|c|c|c|c|c|}
\hline \multirow{2}{*}{ QTL } & \multirow{2}{*}{ Interval } & \multirow{2}{*}{ Year } & \multicolumn{3}{|c|}{$\leqslant 4-27$} & \multicolumn{3}{|c|}{$4-27-5-4$} & \multicolumn{3}{|c|}{$5-4-5-11$} & \multicolumn{3}{|c|}{$5-11-5-18$} & \multicolumn{3}{|c|}{$5-18-5-25$} \\
\hline & & & LOD & $A$ & $R^{2} \%$ & LOD & $A$ & $R^{2} \%$ & LOD & $A$ & $R^{2} \%$ & LOD & $A$ & $R^{2} \%$ & LOD & $A$ & $R^{2} \%$ \\
\hline \multirow[t]{2}{*}{$Q H t-2 B$} & $X w m c 154-X g w m 257$ & 2004 & & & & & & & & & & & & & 3.49 & -1.48 & 7.04 \\
\hline & & 2005 & & & & & & & & & & & & & 2.51 & -1.18 & 4.81 \\
\hline \multirow[t]{2}{*}{$Q H t-3 A$} & Хсаи206-Xgwm383 & 2004 & & & & & & & & & & & & & & & \\
\hline & & 2005 & & & & 2.54 & -1.47 & 10.07 & & & & & & & & & \\
\hline \multirow[t]{2}{*}{$Q H t-3 B$} & Xgwm566-Xcau101 & 2004 & 3.68 & 2.47 & 7.22 & & & & & & & & & & & & \\
\hline & & 2005 & 2.56 & 1.69 & 4.81 & & & & & & & & & & & & \\
\hline \multirow[t]{2}{*}{$Q H t-4 B-1$} & Xgwm375-Xgwm 251 & 2004 & 5.77 & -3.08 & 11.00 & & & & & & & & & & & & \\
\hline & & 2005 & 5.47 & -2.48 & 10.57 & 2.52 & -1.32 & 8.04 & & & & & & & & & \\
\hline \multirow[t]{2}{*}{$Q H t-4 B-2$} & Xgwm495-Xgwm375 & 2004 & & & & 3.81 & -1.73 & 8.00 & & & & 3.37 & -1.38 & 7.99 & & & \\
\hline & & 2005 & & & & & & & & & & 3.52 & -2.75 & 7.84 & 3.21 & -1.60 & 9.07 \\
\hline \multirow[t]{2}{*}{$Q H t-4 D$} & Xpsp3103-Xcfd71 & 2004 & & & & & & & 3.03 & -1.72 & 6.96 & & & & & & \\
\hline & & 2005 & & & & & & & & & & 4.02 & -3.48 & 12.70 & & & \\
\hline \multirow[t]{2}{*}{$Q H t-5 A$} & $Q-X c f a 2155$ & 2004 & & & & & & & & & & & & & 6.39 & -2.07 & 13.95 \\
\hline & & 2005 & & & & & & & & & & 3.27 & -2.88 & 8.28 & 6.63 & -1.93 & 13.07 \\
\hline \multirow[t]{2}{*}{$Q H t-7 B-1$} & Xcau130-Xgwm537 & 2004 & & & & & & & & & & & & & & & \\
\hline & & 2005 & 3.03 & 1.9 & 6.04 & & & & & & & & & & & & \\
\hline
\end{tabular}

a) Same as in Table 2 . 
detected at the first four stages in 2004, but was only detected at the first stage in 2005 by unconditional analysis. However, the locus was detected by conditional analysis at the first stage in both 2004 and 2005, and the effect value in 2004 was larger than in 2005. All these differences observed might result from gene-environment interaction. More importantly, these unstable QTLs might be more sensitive to the environment than QTLs detected at the same stages in the two years.

Flowering time is probably another factor that influences plant height. The flowering time of Altgold (about June 1) was significantly later than that of Line 3338 (about May 10), and the plant height of Altgold was much higher than that of Line 3338 during the heading to flowering period owing to elongation of the uppermost internode (data not shown). In the current study, the locus $Q H t-5 \mathrm{~A}$, with the $q$ allele obtained from Altgold, increased plant height and was expressed exclusively in Altgold after May 18 in 2004 and May 11 in 2005 (Tables 2 and 3) and, as mentioned above, the plant height of Line 3338 remained unchanged after May 11. Thus it could be inferred that this locus was responsible for elongation of the uppermost internode in Altgold after heading and until flowering.

In previous studies, which had the objective of investigating the developmental behavior of quantitative traits, equal intervals in the developmental period were usually employed for conditional analysis [22-24]. In the present study, 7-d intervals from April 27th to May 25th were used for dynamic characterization of plant height QTLs in wheat. At the 5 time points, we detected nine QTLs by the unconditional QTL mapping method, which were located on chromosomes 2D, 4A, 4B, 4D, 5A and 7B, respectively, and 6 of these QTLs were also identified by conditional QTL analysis. In contrast, the other three unconditional QTLs ( $Q H t-2 D, Q H t-4 A$ and $Q H t-7 B-2)$ were not detected by conditional QTL mapping. Moreover, another 5 conditional QTLs (QHt-3A,QHt-3B,QHt-4B,QHt-4D and $Q H t-7 B-1)$ were detected at the 5 investigated developmental stages, but not at the maturing stage, which might reflect that the expression of genes is not uniform among all of the intervals, and the basis for division of measurement stages needs further study. More recently, Wu et al. [31] developed a statistical model, called functional mapping, which was proposed to evaluate QTL values with respect to a plant growth curve, allowing for complete characterization of the network of all possible genes that confer the temporal pattern of variation in a complex dynamic trait. The approach may be beneficial to cope with the problem of division of measurement stages. However, it is important for functional mapping to evaluate the error of estimation for mathematical model parameters in future studies [32].

We also identified two features of expression of plant height QTL in the wheat RIL population: (1) the effect of each QTL/gene was in one direction (with +/- value) and independent of developmental stage; for instance, the un- conditional QTL QHt-3B contributed a positive additive effect at the 4 stages at which it was detected, while the unconditional and conditional $Q H t-4 B-1$ contributed a negative additive effect at all the stages it was expressed; (2) expression of 6 conditional QTLs was in the order $Q H t-3 B \rightarrow Q H t-4 B-1 \rightarrow Q H t-4 B-2 \rightarrow Q H t-4 D \rightarrow Q H t-5 A$ and $Q H t-2 B$ expressed at the same developmental stage, which indicated that expression of QTLs/genes at sequential developmental stages, followed a certain order. The stable temporal order of QTL/gene expression in both years may also imply existence of network of genetic interactions, such as the gibberellin signaling pathway, whose relationship with plant height has been discussed extensively [33].

Twenty-one Rht (Reduced plant height) genes have been identified in wheat thus far [2], among which Rht-Blb (Rhtl) and Rht-Dlb (Rht2), located on chromosomes 4B and $4 \mathrm{D}$, respectively, have been used successfully in wheat breeding programs worldwide. In this study, 2 QTLs $(Q H t-4 B-1$ and $Q H t-4 B-2)$ closely linked to plant height were located on chromosome 4B. In our study, $Q H t-4 B-1$ was located between Xgwm251 and Xgwm375, and $Q H t-4 B-2$ was located in the chromosomal region between Xgwm375 and Xgwm495. In addition, Xgwm251, Xgwm375 and Xgwm149 are reported to be located in the same chromosome bin (4BL1-0.86-1.00) (http://wheat.pw.usda.gov), and another QTL for plant height, which is linked to Xgwm149, has been mapped at the same location by Huang et al. [9]. Furthermore, two molecular markers (Xfbal and Xgwm495) were also reported to be linked to $R h t / B 1$ [12]. More recently, a plant height QTL linked to Xgwm495 was also detected using the DH population derived from RL4452 $\times$ AC Domain [3]. Though Rht/B1 is reported to be located on the short arm of chromosome $4 \mathrm{~B}$, it is quite different from the QTLs (QHt-4B-1 and $Q H t-4 B-2)$ identified in our study that were located on the other arm of this chromosome (Figure 1), suggesting that $Q H t-4 B-1$ and $Q H t-4 B-2$ are new QTLs for plant height. Another dwarfism gene, Rht-D1, mapped on chromosome 4D was closely linked to the marker Xfba211 [12]. In the present study, one QTL $(Q H t-4 D)$ was located on chromosome 4D and was flanked by xpsp3103 and xcfd71. Another two major plant height QTLs $(Q H t-2 B$ and $Q h t-7 B-1)$ detected in this study were reported previously by Gervais et al. [7] and Huang et al. [11], respectively. It was reported also that the marker Xgwm257 was linked closely with the $P p d-B 1$ gene [34]. This marker was linked with $Q H t-2 B$ in our study, suggesting that $Q H t-2 B$ might represent the $P p d-B 1$ gene. Taken together, these QTLs detected by dynamic mapping were highly consistent with that of a final trait phenotype in other populations, and most had a large effect with LOD values ranging from 2.52 to 7.58 . Furthermore, the $Q$ gene is a well-characterized gene that governs the free-threshing character, square spike phenotype, spike length and plant height [27]. A $Q$ gene-specific marker was developed and located on chromosome 5A (Figure 1). Most importantly, 
we detected a QTL $(Q H t-5 A)$ located between $Q$ and xcfa2155 (Figure 1), and the locus was closely linked with $Q$. As expected, the $q$ allele detected in spelt wheat Altgold contributed positively to plant height, while the $Q$ allele in common wheat Line 3338 was negative to the trait (Tables 2 and 3), which is in agreement with a previous study [35].

In conclusion, the developmental behavior of wheat plant height was dissected in this study. QTLs detected by conditional and unconditional analysis in the present study are useful for marker-assisted selection and fine mapping of genes controlling wheat plant height. As for plant height, the development of many other agronomic traits is a dynamic process, and closer attention should be paid to detection of the developmental QTLs of these traits, which can be further used for pyramid breeding by marker-aided selection. Another striking result in the present study was that QTL expression during development of the plant height trait showed selectivity and followed a temporal order, and this may be helpful to dissect the genetic and molecular mechanisms of plant height development.

This work was supported by the National Basic Research Program of China (2001CB108800 and 2007CB109000).

1 Worland A J, Korzun V, Röder M S, et al. Genetic analysis of the dwarfing gene Rht 8 in wheat. Part II. The distribution and adaptive significance of allelic variants at the Rht8 locus of wheat as revealed by microsatellite screening. Theor Appl Genet, 1998, 96: 1110-1120

2 McIntosh R A, Hart G E, Gale M D. Catalogue of gene symbols for wheat. In: Li Z S, Xin Z Y, eds. Proc. 8th Int. Wheat Genet Symp. Beijing: China Agricultural Scientech, 1995. 1333-1500

3 McCartney C A, Somers D J, Humphreys D G, et al. Mapping quantitative trait loci controlling agronomic traits in the spring wheat cross RL4452 × ‘AC Domain'. Genome, 2005, 48: 870-883

4 Börner A, Schumann E, Fürste A, et al. Mapping of quantitative trait loci determining agronomic important characters in hexaploid wheat (Triticum aestivum L.). Theor Appl Genet, 2002, 105: 921-936

5 Ellis M H, Rebetzke G J, Azanza F, et al. Molecular mapping of gibberellin-responsive dwarfing genes in bread wheat. Theor Appl Genet, 2005, 111: 423-430

6 Marza F, Bai G H, Carver B F, et al. Quantitative trait loci for yield and related traits in the wheat population Ning7840 $\times$ Clark. Theor Appl Genet, 2006, 112: 688-698

7 Gervais L, Dedryver F, Morlais J Y, et al. Mapping of quantitative trait loci for field resistance to Fusarium head blight in an European winter wheat. Theor Appl Genet, 2003, 106: 961-970

8 Eriksen L, Borum F, Jahoor A. Inheritance and localization of resistance to Mycosphaerella graminicola causing septoria tritici blotch and plant height in the wheat (Triticum aestivum L.) genome with DNA markers. Theor Appl Genet, 2003, 107: 515-527

9 Huang X Q, Coster H, Ganal M W, et al. Advanced backcross QTL analysis for the identification of quantitative trait loci alleles from wild relatives of wheat (Triticum aestivum L.). Theor Appl Genet, 2003, 106: 1379-1389

10 Verma V, Worland A J, Sayers E J, et al. Identification and characterization of quantitative trait loci related to lodging resistance and associated traits in bread wheat. Plant Breed, 2005, 124: 234-241

11 Huang X Q, Cloutier S, Lycar L, et al. Molecular detection of QTLs for agronomic and quality traits in a doubled haploid population derived from two Canadian wheats (Triticum aestivum L.). Theor Appl Genet, 2006, 113: 753-766

12 Cadalen T, Sourdille P, Charmet G, et al. Molecular markers linked to genes affecting plant height in wheat using a doubled-haploid population. Theor Appl Genet, 1998, 96: 933-940

13 Liu Z H, Anderson J A, Hu J, et al. A wheat intervarietal genetic linkage map based on microsatellite and target region amplified polymorphism markers and its utility for detecting quantitative trait loci. Theor Appl Genet, 2005, 111: 782-794

14 Ayala L, Henry M, van Ginkel M, et al. Identification of QTLs for BYDV tolerance in bread wheat. Euphytica, 2002, 128: 249-259

15 Peng J H, Ronin Y, Fahima T, et al. Domestication quantitative trait loci in Triticum dicoccoides, the progenitor of wheat. Proc Natl Acad Sci USA, 2003, 100: 2489-2494

16 Kherialla A I, Whittington A J. Genetic analysis of growth in tomato: The $\mathrm{F}_{1}$ generation. Ann Bot, 1962, 26: 489-504

17 Peat W E, Whittington A J. Genetic analysis of growth in tomato: Segregation generation. Ann Bot, 1965, 29: 725-738

$18 \mathrm{Wu} \mathrm{K} \mathrm{H}$. Analysis of gene effects for three quantitative characters at different development stages in maize. Acta Genet Sin, 1987, 14: 363-369

$19 \mathrm{Xu} \mathrm{Y} \mathrm{B,} \mathrm{Shen} \mathrm{Z} \mathrm{T.} \mathrm{Diallel} \mathrm{analysis} \mathrm{of} \mathrm{tiller} \mathrm{number} \mathrm{at} \mathrm{different}$ growth stages in rice (Oryza sativa L.). Theor Appl Genet, 1991, 3: 243-249

20 Zhu J. Methods of predicting genotype value and heterosis for offspring of hybrids. J Biomath, 1993, 8: 32-44

21 Zhu J. Analysis of conditional genetic effects and variance components in developmental genetics. Genetics, 1995, 141: 1633-1639

22 Yan J Q, Zhu J, He C X, et al. Molecular dissection of developmental behavior of plant height in rice (Oryza sativa L). Genetics, 1998, 150: 1257-1265

23 Yan J B, Tang H, Huang Y Q, et al. Dynamic analysis of QTL for plant height at different developmental stages in maize (Zea mays L.). Chinese Sci Bull, 2003, 48: 2601-2607

24 Sun D S, Li W B, Zhang Z C, et al. Analysis of QTL for plant height at different developmental stages in soybean. Acta Agronom Sin, 2006, 32: 509-514

25 Wang Z H, Wu X S, Ren Q, et al. QTL mapping for developmental behavior of plant height in wheat (Triticum aestivum L.). Euphytica, 2010, 174: 447-458

26 Campbell K G. Spelt: Agronomy, genetics, and breeding. Plant Breed Rev, 1997, 15: 187-213

27 Liu D C, Gao M Q, Guan R X, et al. Mapping quantitative trait loci for plant height in wheat (Triticum aestivum $\mathrm{L}$.) using a $\mathrm{F}_{2: 3}$ population. Acta Genet Sin, 2002, 29: 706-711

28 Simons K J, Fellers J P, Trick H N, et al. Molecular characterization of the major wheat domestication gene Q. Genetics, 2006, 172: 547- 555

29 Ellis M H, Spielmeyer W, Gale K R, et al. "Perfect" markers for the Rht-B1b and Rht-D1b dwarfing genes in wheat. Theor Appl Genet, 2002, 105: 1038-1042

30 Zeng Z B. Precision mapping of quantitative trait loci. Genetics, 1994, 136: 1457-1468

31 Wu R, Li M. Functional mapping-How to map and study the genetic architecture of dynamic complex traits. Nat Rev Genet, 2006, 7 : 229-237

32 Zhang Y M. Research progress of QTL mapping method in crop. Chinese Sci Bull, 2006, 51: 2223-2231

33 Hartweck L M. Gibberellin signaling. Planta, 2008, 229: 1-13

34 Mohler V, Lukman R, Ortiz-Islas S, et al. Genetic and physical mapping of photoperiod insensitive gene $P p d-B 1$ in common wheat. Euphytica, 2004, 138: 33-40

35 Kato K, Sonokawa R, Miura H. Dwarfing effect associated with the threshability gene Q on wheat chromosome 5A. Plant Breed, 2003, 122: 489-492

Open Access This article is distributed under the terms of the Creative Commons Attribution License which permits any use, distribution, and reproduction in any medium, provided the original author(s) and source are credited. 